ARTICLE

\title{
Self-templated synthesis of uniform hollow spheres based on highly conjugated three-dimensional covalent organic frameworks
}

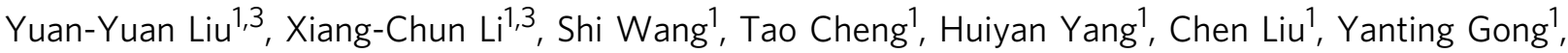

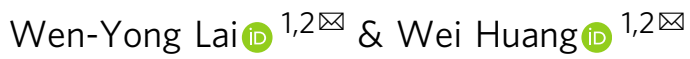

Covalent organic frameworks (COFs) have served as a family of porous crystalline molecules for various promising applications. However, controllable synthesis of COFs with uniform morphology is paramount yet still remains quite challenging. Herein, we report self-templated synthesis of uniform and unique hollow spheres based on highly conjugated threedimensional (3D) COFs with diameters of $500-700 \mathrm{~nm}$. A detailed time-dependent study reveals the continuous transformation from initial nano sphere-like particles into uniform hollow spherical structures with Ostwald ripening mechanism. Particularly, the resulting 3D COF (3D-Sp-COF) is prone to transport ions more efficiently and the lithium-ion transference number $\left(t^{+}\right)$of 3D-Sp-COF reaches 0.7 , which even overwhelms most typical PEO-based polymer electrolytes. Inspiringly, the hollow spherical structures show enhanced capacitance performance with a specific capacitance of $251 \mathrm{~F} \mathrm{~g}^{-1}$ at $0.5 \mathrm{~A} \mathrm{~g}^{-1}$, which compares favorably with the vast majority of two-dimensional COFs and other porous electrode materials.

\footnotetext{
${ }^{1}$ Key Laboratory for Organic Electronics and Information Displays \& Institute of Advanced Materials (IAM), Nanjing University of Posts \& Telecommunications, 9 Wenyuan Road, 210023 Nanjing, China. ${ }^{2}$ Frontiers Science Center for Flexible Electronics (FSCFE), MIIT Key Laboratory of Flexible Electronics (KLoFE), Northwestern Polytechnical University, West Youyi Road, 710072 Xi'an, China. ${ }^{3}$ These authors contributed equally: Yuan-Yuan Liu,

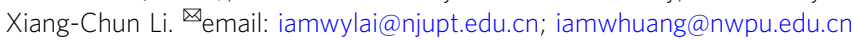


C ovalent organic frameworks (COFs), as an important type of crystalline porous materials with well-defined and predictable structures, demonstrate extensive applications in molecule storage and separation ${ }^{1,2}$, catalysis ${ }^{3,4}$, energy storage $^{5,6}$, optoelectronics ${ }^{7,8}$, and sensors ${ }^{9,10}$. Despite their crystalline features, COFs are usually obtained as microcrystalline morphologies and their long-range ordered growth is limited in the nano/micro domain because of the internal defects and kinetic trapping of smaller crystallites ${ }^{11-13}$. The morphology of the crystallites is an important factor for their applications, such as molecular absorption and catalysis ${ }^{14,15}$, drug delivery ${ }^{16,17}$, energy storage ${ }^{18,19}$, etc. In general, the geometry of the building blocks plays a key role to determine the morphology of the crystallites $^{20-22}$. Based on the geometry of the building blocks, COFs are mainly classified as two-dimensional (2D) COFs and three-dimensional (3D) $\mathrm{COFs}^{23-25}$. Since the first report on 2D COFs presented by Yaghi and co-workers in 2005 (ref. ${ }^{26}$ ), extensive efforts have been devoted to this fast developing area $6,27,28$. Controllable synthesis of COF crystallites with uniform morphology, which is beneficial to fine modulating the material performance and better understanding the forming mechanisms of COF crystallites ${ }^{29,30}$, is highly desirable yet quite challenging ${ }^{11,31,32}$. Various COFs with uniform morphology such as free-standing films $s^{33-36}$, nanosheets ${ }^{37-39}$, tubes ${ }^{12,40}$, shuttle ${ }^{41}$, belts $^{22,42}$, fibers ${ }^{43,44}$, and spheres ${ }^{45,46}$ have been recently reported mainly based on $2 \mathrm{D}$ COFs with fully exploring the potential of the 2D-layered structures. As compared with 2D COFs, 3D COFs with extended networks are generally able to achieve intrinsically large surface areas ${ }^{47,48}$, low density of open sites ${ }^{49,50}$, and fascinating confinement effects ${ }^{51-53}$, which have recently received increasing interest. However, the synthesis of 3D COFs with uniform morphologies remains unexplored. It is quite challenging to obtain 3D COF crystallite structures with uniform morphology mainly due to the "unavoidable" existence of cross-linked networks ${ }^{53}$. Considering their unique chemical and structural characteristics, it is charming to construct 3D COFs with uniform morphologies via controllable synthesis.

Herein, we report the controllable synthesis of 3D COFs (3D-Sp-COF) with uniform and unique hollow spherical morphology based on highly conjugated building blocks via self-templated synthetic methods. Their formation mechanism of hollow spheres based on 3D COFs has been investigated by a detailed time-dependent study and the growth process has been dominated by Ostwald ripening. Interestingly, all the 3D COFs show large surface area (up to $1016 \mathrm{~m}^{2} \mathrm{~g}^{-1}$ ) and high ion mobility, which serve as attractive candidates as electrode materials for energy storage applications. Among these, the well-defined hollow spherical 3D-Sp-COF@96h shows enhanced specific capacitance $\left(251 \mathrm{Fg}^{-1}\right.$ at $\left.0.5 \mathrm{Ag}^{-1}\right)$ as compared with the $3 \mathrm{D}$ COFs with defective intermediate morphology, revealing that the characteristics of $3 \mathrm{D}$ COFs can be facilely regulated by exploring the potential of the microstructures. According to our preliminary specific capacitance studies, overwhelming high specific capacitance of $251 \mathrm{Fg}^{-1}$ has been achieved for $3 \mathrm{D}$ COFs by facilely modulating the morphological features.

\section{Results}

Synthesis and structural characterizations. Schematic illustration for the synthesis of $3 \mathrm{D}-\mathrm{Sp}-\mathrm{COF}$ is depicted in Fig. 1a. Spirobifluorene core $\mathrm{Sp}-4\left(\mathrm{Ph}-\mathrm{NH}_{2}\right)$ was synthesized by Suzuki cross-coupling reaction (Supplementary Figs. 1-4). 3D COF (3D$\mathrm{Sp}-\mathrm{COF}$ ) with spirobifluorene core and imine bonds was prepared by the solvothermal reaction between $\mathrm{Sp}-4\left(\mathrm{Ph}-\mathrm{NH}_{2}\right)$ and terephthalaldehyde $(\mathrm{Ph}-2 \mathrm{CHO})$ with an acetic acid catalyst at $120^{\circ} \mathrm{C}$ (Supplementary Fig. 5). For comparison, polymerization between $\mathrm{Sp}-4\left(\mathrm{Ph}-\mathrm{NH}_{2}\right)$ and 2,5-dihydroxyterephthalaldehyde was also conducted under the same conditions to afford 3DSpOH-COF (Supplementary Fig. 5). 3D-Sp-COF and 3D-SpOHCOF were isolated as yellow microgranular powder in $72 \%$ and orange powder in $68 \%$ yield, respectively. The crystalline nature of 3D-Sp-COF was determined by powder X-ray diffraction (PXRD) (Fig. 1b). As shown in the experimental PXRD pattern (Fig. 1b, black curve), 3D-Sp-COF exhibited intense diffraction peaks $(2 \theta)$ at $4.73^{\circ}, 9.52^{\circ}, 12.36^{\circ}$, and $14.13^{\circ}$ corresponding to the (200), (400), (411), and (501) Bragg planes of the space group I41/AMD, respectively, indicating high crystallinity of the resulting 3D COF. 3D-SpOH-COF exhibited intense diffraction peaks $(2 \theta)$ at $4.17^{\circ}, 8.46^{\circ}, 10.07^{\circ}, 12.76^{\circ}, 13.92^{\circ}, 19.78^{\circ}$, and $14.13^{\circ}$ corresponding to the (200), (400), (211), (411), (431), and (631), respectively (Supplementary Figs. 6 and 7). Pawley refined PXRD patterns (Supplementary Fig. 6, red) with negligible difference (residuals $R_{\mathrm{p}}=6.58 \%, R_{\mathrm{wp}}=12.18 \%$ for $3 \mathrm{D}$-Sp-COF and residuals $R_{\mathrm{p}}=6.53 \%, R_{\mathrm{wp}}=11.75 \%$ for $3 \mathrm{D}-\mathrm{SpOH}-\mathrm{COF}$ ) compared favorably with the experimental patterns, which shows that the obtained crystal structure is very close to the predicted structure. To illustrate the lattice packing, the crystal models were constructed by employing Materials Studio 8.0 software package. Considering that 3D-Sp-COF was composed of tetragonal disphenoid-shaped spirobifluorene core and linear imine bonds, the possible lattice structures with different interpenetration degrees were proposed to demonstrate a fold-interpenetrated diamond (dia) topology similar to the reported SP-3D-COF 1 and SP-3D-COF 2 (ref. ${ }^{53}$ ). According to the detailed simulation, the calculated PXRD pattern of 3D-Sp-COF (Fig. 1b, red curve) of sevenfold-interpenetrated diamond (dia-c7) net with I41/AMD symmetry was consistent with the experimental results. The refinement data of 3D-Sp-COF $(a=b=35.554 \AA, c=11.351 \AA$, $\left.\alpha=\beta=\gamma=90.0^{\circ}\right)$ and 3D-SpOH-COF $(a=b=41.6510 \AA, c=$ $9.9993 \AA, \alpha=\beta=\gamma=90.0^{\circ}$, Fig. S5) both yielded a unit cell with parameters nearly equivalent to those of suggested models. The other possible lattice structures with non-, twofold-, threefold-, fourfold-, fivefold-, and sixfold-interpenetrated diamond topologies for 3D-Sp-COF were constructed with different space groups (Supplementary Figs. 8-14). However, the simulated PXRD patterns of these hypothetical topologies were not in agreement with the experimental results. Obviously, the lattice structures of 3D$\mathrm{Sp}-\mathrm{COF}$ and 3D-SpOH-COF (dia-c7) are different from those of SP-3D-COF 1 (dia-c6 $)^{53}$, which may play a role to affect the micro-morphology of the resulting COFs.

To elucidate the Schiff-base polymerization, Fourier transform infrared spectra of Sp-4(Ph- $\left.\mathrm{NH}_{2}\right), \mathrm{Ph}-2 \mathrm{CHO}$, and 3D-Sp-COF were tested (Supplementary Fig. 15). The aldehyde band at 1703 $\mathrm{cm}^{-1}$ and the $\mathrm{N}-\mathrm{H}$ stretching band at $3372 \mathrm{~cm}^{-1}$ were significantly weakened after the Schiff-base reaction. However, the new $\mathrm{C}=\mathrm{N}$ stretching band at $1625 \mathrm{~cm}^{-1}$ was significantly enhanced, which suggested the formation of the imine linkage in this $3 \mathrm{D}$ COF. Note that the $\mathrm{C}=\mathrm{O}$ stretching band at $1703 \mathrm{~cm}^{-1}$ appeared in $3 \mathrm{D}-\mathrm{Sp}-\mathrm{COF}$, probably due to moisture included in the pores. According to thermogravimetric analysis, 3D-Sp-COF showed an excellent thermal stability up to $475^{\circ} \mathrm{C}$ (Supplementary Fig. 16). As shown in Fig. 2, 3D-Sp-COF is prone to transport ions (such as $\mathrm{Li}^{+}$) more efficiently. As predicted, the lithium-ion transference number $\left(t^{+}\right)$of $3 \mathrm{D}-\mathrm{Sp}$-COF of 0.7 is recorded (Supplementary Table 1), which overwhelms most typical polyethylene oxide (PEO)-based polymer electrolytes 54,55 and even comparable to the signal-ion electrolyte systems ${ }^{56-58}$. According to the results, ion conduction pathways provided by $3 \mathrm{D}$ COFs play a positive role on improving the ion transport. The lithium ion cell has a discharge capacity of 130,120 , and $75 \mathrm{~mA} \mathrm{~h}$ $\mathrm{g}^{-1}$ at the current densities of 54,105 , and $210 \mu \mathrm{A}$, respectively, 

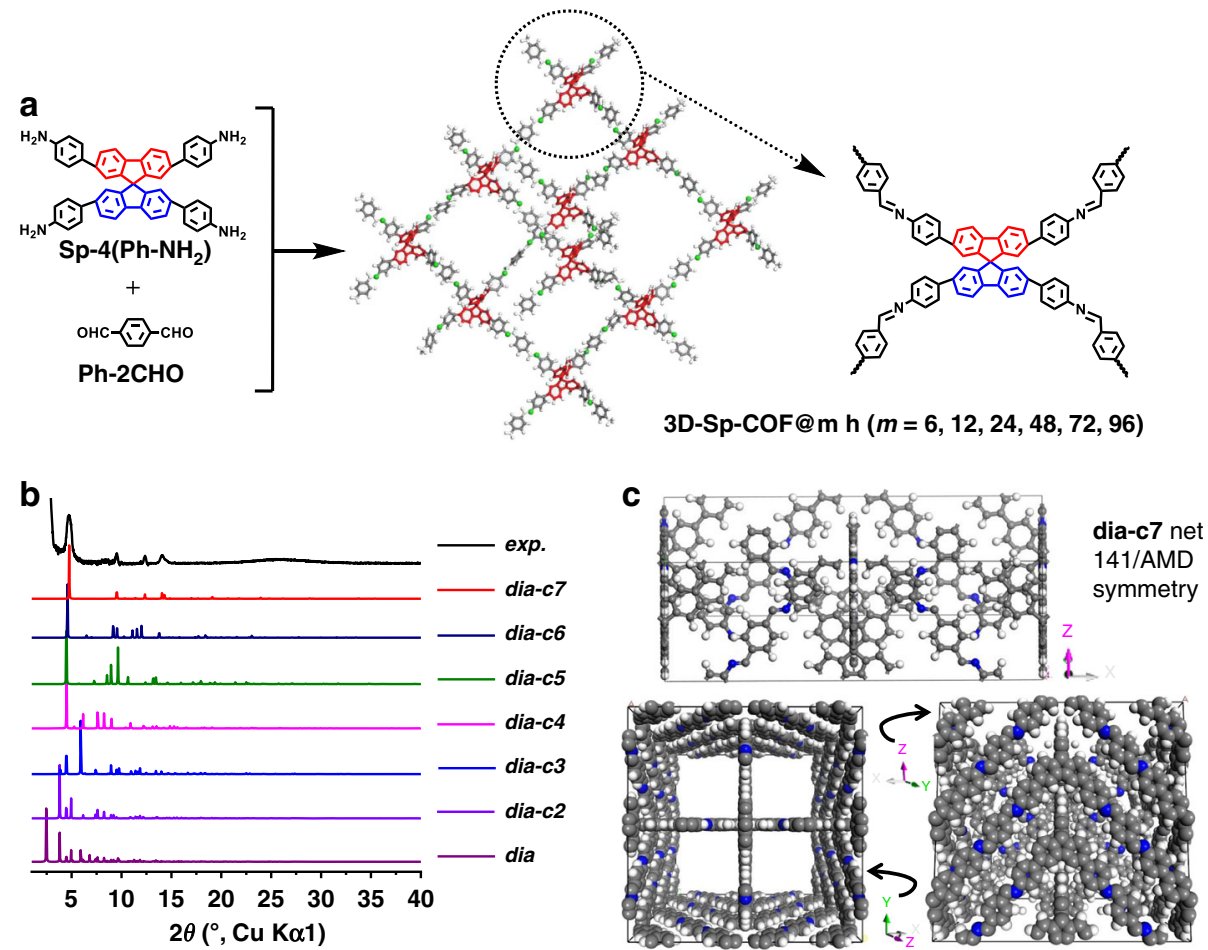

Fig. 1 Synthesis and characterization of 3D-Sp-COFs. a Schematic illustration for the synthesis of 3D-Sp-COF (@m h is on behalf of the reaction time). b The experimental PXRD profiles of 3D-Sp-COF (black curve) compared with theoretical PXRD calculated from crystal models with different degrees of interpenetration. c Unit cell, top view, and side view of the cell of 3D-Sp-COF (dia-c7): C, gray; N, blue; H, white.

a

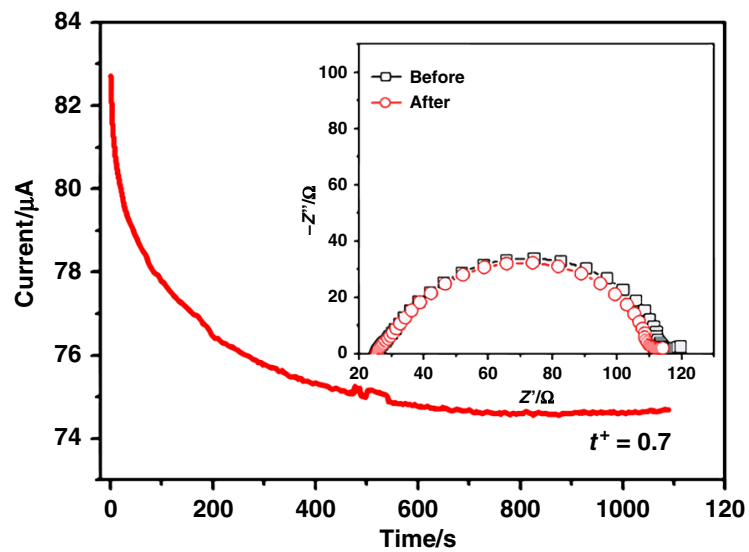

b

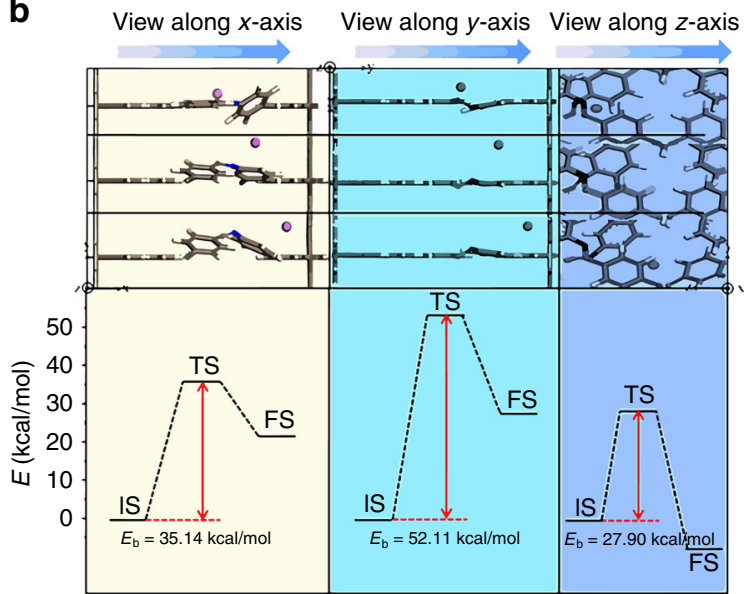

Fig. 2 The lithium ion migration characterization of 3D-Sp-COF. a The chronoamperometry of the Li/3D-Sp-COF/Li symmetric cells (experiment condition: ambient temperature, potential step of $10 \mathrm{mV}$ ). The insets present the ElS before and after polarization at ambient temperature. $\mathbf{b}$ Theoretical calculation of Li-ion migration behaviors inside the pore (top) with corresponding energy diagrams (bottom). The initial, transition, and final states are abbreviated as IS, TS, and FS, respectively.

suggesting superior cell performance using 3D-Sp-COF-based electrolyte (Supplementary Fig. 17). The lithium ion migration phenomenon of $3 \mathrm{D}-\mathrm{Sp}-\mathrm{COF}$ is explained by density functional theory calculation. Specifically, lithium ion migration in the pores is studied by comparing three pathways ( $x$-axis pathway, $y$-axis pathway, and $z$-axis pathway; Supplementary Fig. 18). The results show that the Li-ion migration along the $x$-axis pathway and $z$ axis pathway require a lower energy barrier (Fig. $2 b$ and Supplementary Table 2) as compared to that along the $y$-axis pathway. This preferred $x$ - and $z$-axial Li-ion migration could be attributed to the shorter hopping distances, which is promoted by the $\mathrm{N}$ atoms of Schiff-base.
Morphology characterizations. Interestingly, well-defined hollow spherical structures were observed for 3D-Sp-COF with an average diameter of 500-700 $\mathrm{nm}$ (Supplementary Fig. 19). To the best of our knowledge, this is the first hollow spheres based on 3D COFs. Similarly, 3D-SpOH-COF also forms well-defined spherical structure (Supplementary Fig. 20). Unlike hollow sphere structure of 3D-Sp-COF, 3D-SpOH-COF shows a solid sphere structure even with reaction time over $96 \mathrm{~h}$ (Supplementary Fig. 20b, 3D-SpOH-COF@96 h). In contrast, although SP-3DCOF 1 with similar spirobifluorene backbone structures shows the characteristics of crystallization, it exists in the form of irregular microcrystals ${ }^{53}$, whereas its long-range ordering growth in 


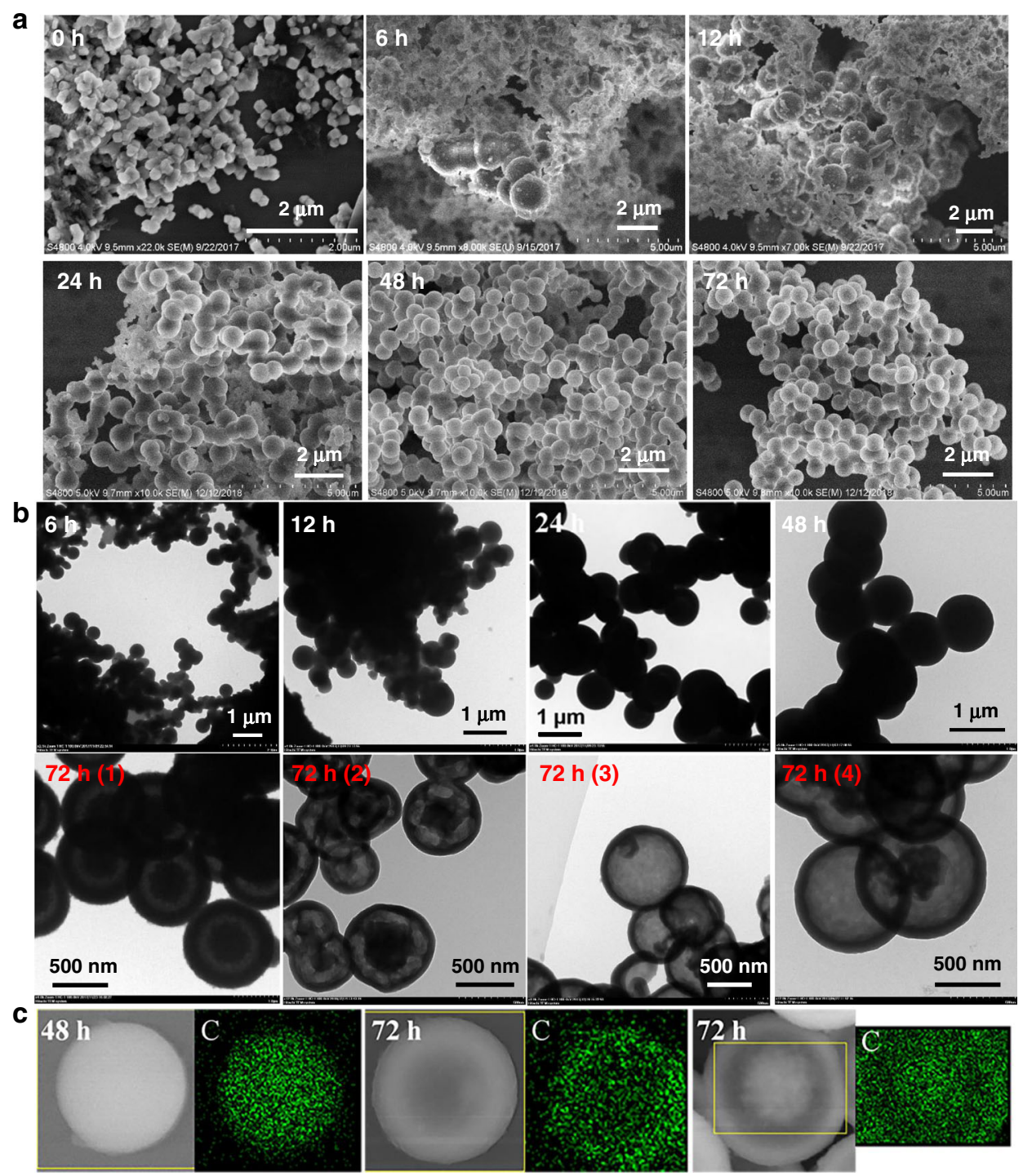

Fig. 3 Structural characterization of 3D-Sp-COFs by different microscopic techniques. a FE-SEM images. b TEM images, 72 h (1)-(4) represents 3D-SpCOF@72 h synthesized from different experimental batches. c SEM-energy-dispersive spectrum (EDS) elemental mapping images: solid sphere (left), full hollowing sphere (middle), and homogeneous core-shell sphere (right).

the nano/microdomain may have been limited due to the internal defects and kinetic trapping of smaller grains. The results suggest that the formation of spherical structure is closely related to chemical structures of the monomers, the reaction conditions, the type of unit cells, and the symmetry groups. To understand the formation mechanism of hollow spheres, the morphological changes at different reaction time $(6,12,24,48,72$, and $96 \mathrm{~h})$ were studied by means of field emission scanning electron microscopy, transmission electron microscopy (TEM), and SEMenergy-dispersive spectrum (EDS) mapping (Fig. 3 and Supplementary Fig. 21). As shown in Fig. 3a, these nanospheres grew up gradually and formed uniform spherical structures with diameters of $500-700 \mathrm{~nm}$ as the reaction time increased. The rough surface on the wall of these nanospheres gradually became smooth. The size of the spheres increased obviously at the beginning of the reaction. After $24 \mathrm{~h}$, the spheres size increased slowly and approximately keeps almost consistent with the increase of reaction time. It is important to note that the structure tend to be strong and stable only when the reaction time is long enough $(>48 \mathrm{~h})$. Otherwise, the structure will collapse during the washing process. As shown in Supplementary Fig. 22, the structure of 3DSp-COF@12 h was decomposed from nanopheres into irregular blocks after repeated washing. Furthermore, TEM images provided useful information to explore the growth pattern of these hollow spheres (Fig. 3b). It was found that 3D-Sp-COF@72h from different experiment batches presented hollow spherical morphologies such as complete hollow spheres, symmetric core-shell hollow spheres, and asymmetric core-shell hollow spheres, which may originate from the packing of original nanoparticles and ripening characteristics. 3D-Sp-COF was also tested after soaking in different solvents for $2 \mathrm{~h}$. Impressively, the crystal form and spherical structures of 3D-Sp-COF remained basically unchanged after treating by different solvents, which shows that 3D-Sp-COF has a good chemical stability (Supplementary Fig. 23). Various hollow spherical structures were also measured by SEM-EDS mapping (Fig. 3c). Based on these data, the construction process of these hollow spherical structures is similar to those of inorganic and organic hollow nanostructures, in which Ostwald ripening dominated the growth of spherical structures $^{59-61}$. 


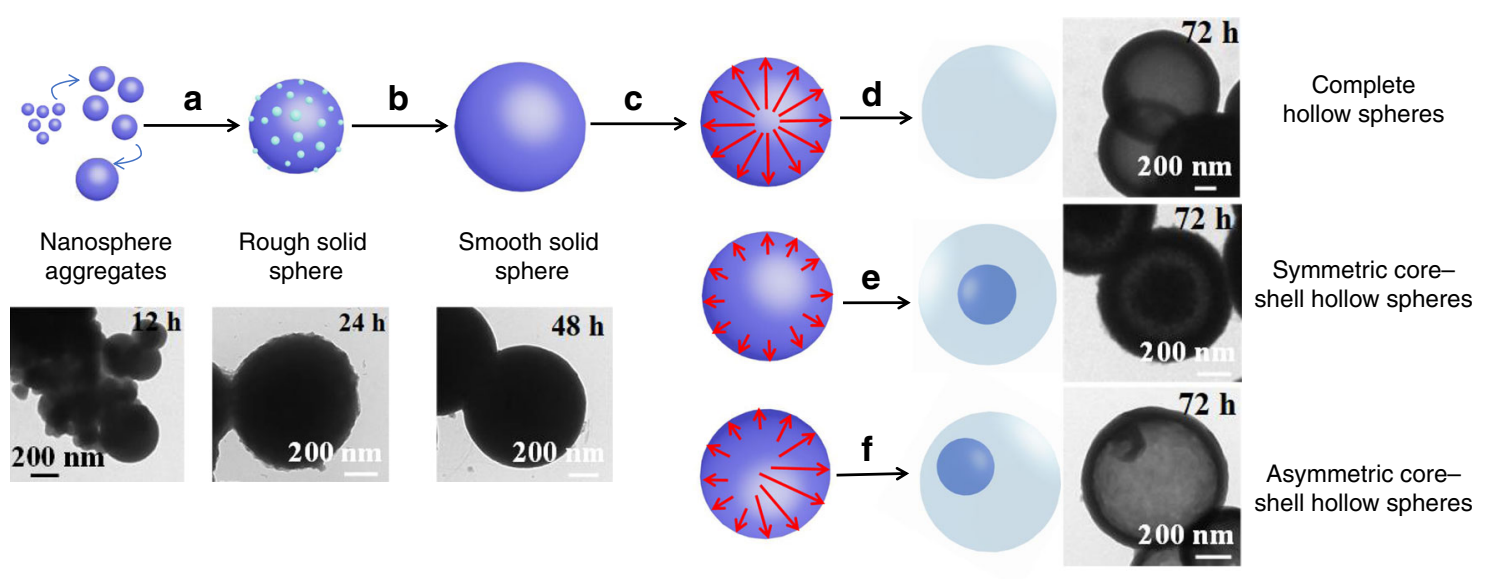

Fig. 4 Self-templated hollowing evolvement process of 3D-Sp-COFs via Ostwald ripening mechanism. a Extinction of small particles and growth of large particles and continuous growth of large particles with dense cores and rough surfaces; $\mathbf{b}$ crystallites on the surface to achieve a smooth morphology; $\mathbf{c}$ dissolution and outmigration of inner crystallites; $\mathbf{d}$ formation of complete hollow spheres; e formation of symmetric core-shell hollow spheres; $\mathbf{f}$ formation of asymmetric core-shell hollow spheres.
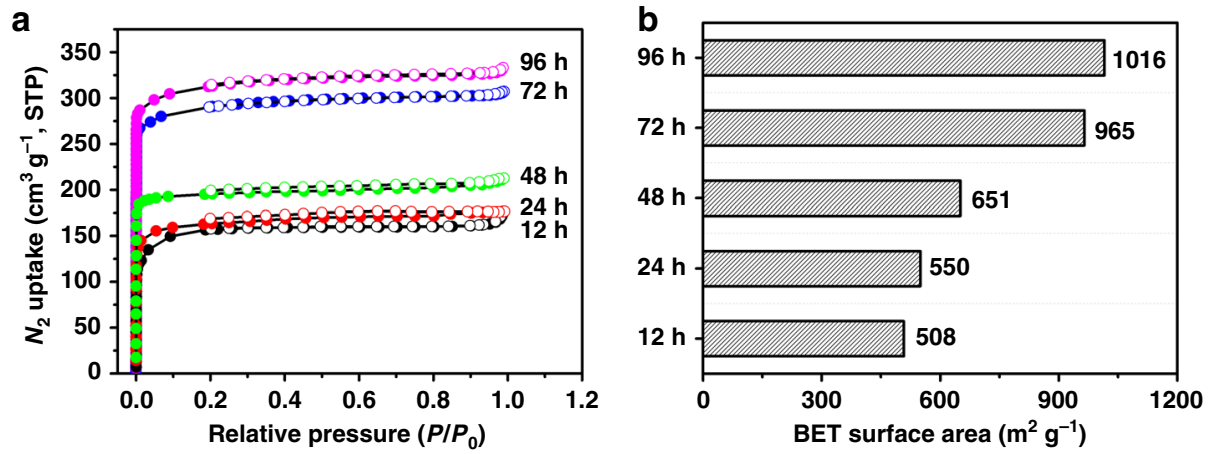

Fig. 5 The porosity of 3D-Sp-COFs. a $N_{2}$ adsorption-desorption isotherms of 3D-Sp-COF@m $\mathrm{h}(m=12,24,48,72,96)$. b The Brunauer-Emmett-Teller (BET) surface area of 3D-Sp-COF-3@m h $(m=12,24,48,72,96)$.

Mechanism of hollow sphere formation. Detailed Ostwaldripening mechanism of hollow spheres based on 3D-Sp-COF is illustrated in Fig. 4. Firstly, due to the Schiff-base polymerization, a large number of small 3D COF microcrystals nucleate from solution and rapidly assemble into larger solid microspheres to reduce the surface energy. Subsequently, the size and density of the solid microspheres continue to grow. In the process of solid growth, the crystal structure on the spherical surface can facilitate the formation of nanoparticles outside the spheres to produce coarse microspheres (Fig. 4a). Next, the surfaces of these crystal microspheres gradually become smooth due to the presence of unreacted amino or aldehyde end functional groups (Fig. 4b). Meanwhile, the crystallization of 3D COF microspheres yielded hollow shell microspheres and microporosity via inside-out Ostwald-ripening mechanism (Fig. 4c). In this case, microspheres finally evolve into complete hollow spheres (Fig. 4d), symmetric core-shell spheres (Fig. 4e), and asymmetric core-shell spheres (Fig. 4f). As shown in Fig. 4, the complete hollow spheres evolve from the center of solid microspheres. The evacuation of symmetric core-shell spheres and asymmetric core-shell spheres begins at the surface of the solid particles ${ }^{62}$. Since the asymmetric Ostwald ripening occurs in the area with lower crystal density, the solid microspheres evolve into asymmetric core-shell spheres or more complex morphologies ${ }^{63}$.

Gas adsorption. The permanent porosity of $3 \mathrm{D}-\mathrm{Sp}-\mathrm{COF}$ at various reaction time was characterized by the nitrogen adsorption-desorption isotherms at $77 \mathrm{~K}$ (Supplementary Figs. 24-28). As shown in Fig. 5a, all 3D-Sp-COFs showed a type $\mathrm{I}$ isotherm with significant increase under low relative pressure region $\left(P / P_{0}<0.05\right)$, demonstrating as a typical property of microporous molecule. The nonlocal density functional theory showed pore size distributions with a major pore width of 1.86 $\mathrm{nm}$ for 3D-Sp-COF@72 h and 3D-Sp-COF@96 h (Supplementary Fig. 29). What is more, the Brunauer-Emmett-Teller (BET) surface area of 3D-Sp-COFs increased with the increment of reaction time. The BET surface area were calculated as 508,550 , 651,965 , and $1016 \mathrm{~m}^{2} \mathrm{~g}^{-1}$ for $12,24,48,72$, and $96 \mathrm{~h}$, respectively. Meanwhile, the total pore volume $\left(V_{\mathrm{P}}\right)$ increased from 0.24 to $0.47 \mathrm{~cm}^{3} \mathrm{~g}^{-1}\left(P / P_{0}=0.99\right)$. Based on these data, the specific surface area of hollow microspheres increased significantly compared with that of the solid microspheres.

Electrochemical performance. Inspired by our previous work ${ }^{64-}$ 66 , the large surface area and conjugated microporous property of 3D-Sp-COFs suggest potential applications in supercapacitance. Cyclic voltammetry (CV) and galvanostatic charge-discharge (GCD) experiments for 3D-Sp-COFs were performed to explore the electrochemical characteristics (Supplementary Figs. 30-35). The reversible redox peaks in the CV plots indicate the specific capacitance is pseudocapacitive in nature. The area under the $\mathrm{CV}$ curve of 3D-Sp-COF@96h with well-defined hollow spherical structure is significantly larger than that of 3D-Sp-COF@6 h for nanoparticles, indicating that the uniform hollow spherical 

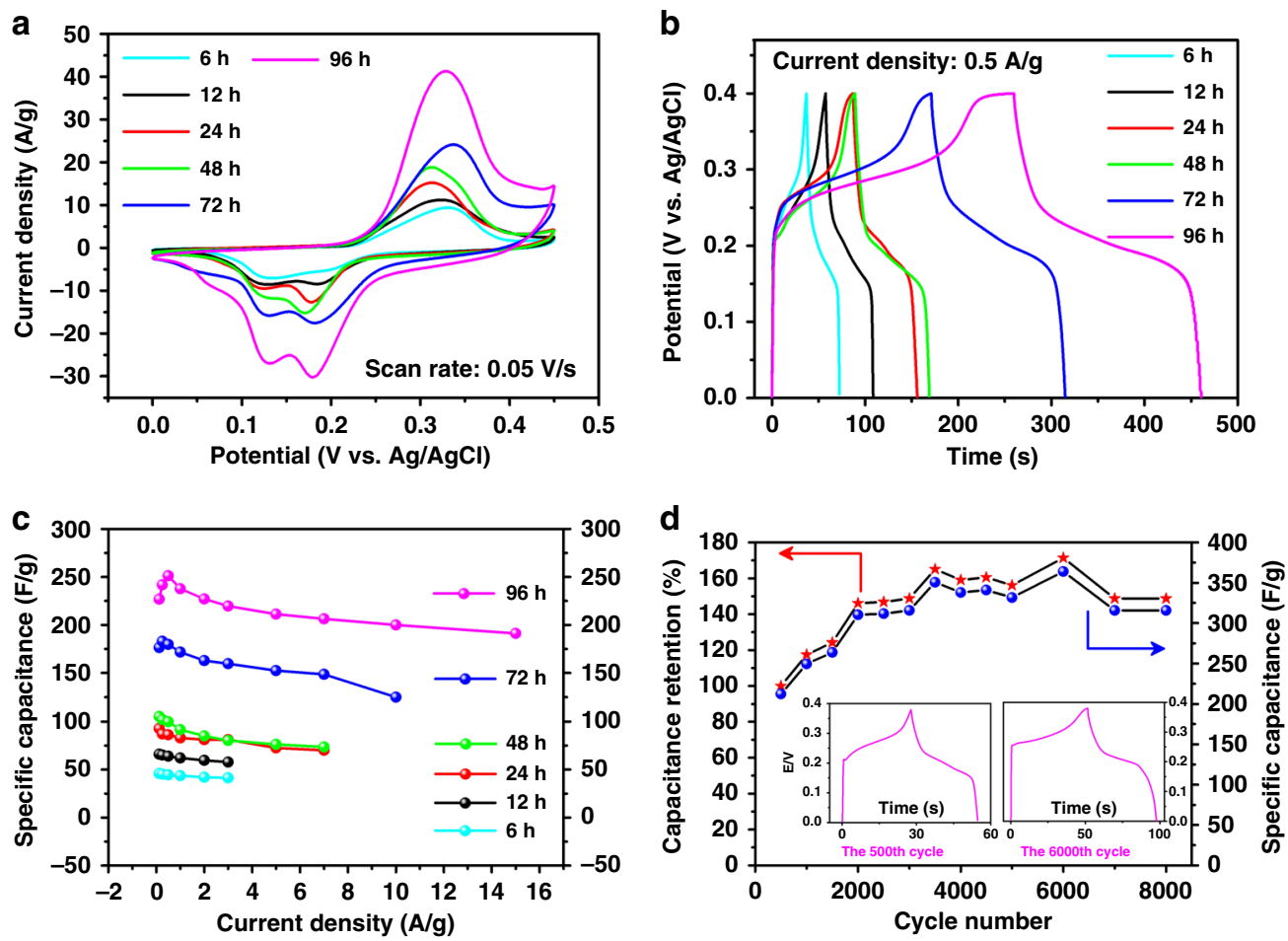

Fig. 6 Electrochemical performance of 3D-Sp-COFs. a CVs at $50 \mathrm{mV} \mathrm{s}^{-1}$. b GCDs at $0.5 \mathrm{Ag}^{-1}$. c Specific capacitance dependence on current density. d Long-term cyclic stability performance of 3D-Sp-COF@96 h at $3 \mathrm{Ag}^{-1}$ in $6 \mathrm{M} \mathrm{KOH}$ electrolyte. Inset: GCD curves of the 500 th and the 6000 th cycle, respectively.

structures are beneficial to improving the capacitance performance (Fig. 6a). It was further verified by GCD test (Fig. 6b). The capacitance performance increases with the increment of reaction time, matching well with $\mathrm{CV}$ data mentioned above. The calculated specific capacitances for 3D-Sp-COF@6h, 3D-Sp-COF@12 h, 3D-Sp-COF@24h, 3D-Sp-COF@48 h, 3D-Sp-COF@72 h, and 3D-Sp-COF@96 $\mathrm{h}$ are 45, 64, 86, 100, 180, and $251 \mathrm{Fg}^{-1}$, respectively, at current densities of $0.5 \mathrm{~A} \mathrm{~g}^{-1}$. Most notably, the specific capacitance of uniform hollow spheres 3D-Sp-COF@96 h is more than twice that of the solid spheres 3D-Sp-COF@48 h. As far as we are concerned, the excellent capacitive value of hollow spheres based on $3 \mathrm{D}-\mathrm{Sp}-\mathrm{COF}$ is superior to that of $2 \mathrm{D}$ COFbased materials previously reported (Supplementary Table 3). Moreover, the capacitance performance overwhelms those of porous electrode molecules, including metal organic frameworks, conjugated microporous polymers, porous triazine-based frameworks, and N-doped carbon nanotubes (Supplementary Table 4) 67-69. In order to study the capacitance stability at high power density, the specific capacitances at different current densities (0.125-15 $\mathrm{A} \mathrm{g}^{-1}$ ) were tested (Fig. 6c). The specific capacitance of 3D-Sp-COF@96 h maintained above $191 \mathrm{~F} \mathrm{~g}^{-1}$ at a high current density of $15 \mathrm{Ag}^{-1}$, implying that such excellent capacitance behaviors can still remained at high power density due to the stable hollow spherical structures. Subsequently, the cycling stability of 3D-Sp-COF@96 h was investigated at current density of $3 \mathrm{~A} \mathrm{~g}^{-1}$ (Fig. 6d). Unexpectedly, the specific capacitance increased dramatically with the increase of cycle numbers, reaching a maximum of $364 \mathrm{~F} \mathrm{~g}^{-1}$ at the 6000th cycle. Even after 8000 cycles, the specific capacitance remained at $316 \mathrm{Fg}^{-1}$, which was 1.4 times as large as the initial value. The increase of specific capacitance during cyclic scanning may be ascribed to the wetting activation of the hollow spheres, which leads to the effective transport of electrolyte ions in the ordered channels. The electron and ion transport characteristics of the 3D-Sp-COF@96h were further studied by electrochemical impedance spectroscopy
(Supplementary Fig. 36). The Nyquist curve shows a steep straight line in the low-frequency region with a small intercept, indicating ideal capacitance behaviors. In addition, there is almost no semicircle in the high frequency region, suggesting that its conductivity is relatively ideal.

\section{Discussion}

In summary, a set of highly conjugated 3D-Sp-COFs was designed and synthesized, beginning from tetrahedral tetraamine and terephthalaldehyde with spirobifluorene core structure as building blocks by means of imine condensation reactions. PXRD patterns and modeling studies demonstrated these 3D COFs are seven-fold-interpenetrated microporous diamond (dia-c7) net with I41/AMD symmetry. Remarkably, 3D-Sp-COF crystallites self-assembled into uniform hollow microspheres with narrow size distribution $(500-700 \mathrm{~nm})$ and large surface area $\left(1016 \mathrm{~m}^{2} \mathrm{~g}\right.$ ${ }^{-1}$ ), which were proved by SEM, TEM, EDS, and BET investigations. The mechanism of hollow spheres formation was studied using a detailed real-time tracking method, indicating that the formation of hollow spheres is the result of Ostwald ripening mechanism. In the $\mathrm{Li} / 3 \mathrm{D}-\mathrm{COF} / \mathrm{Li}$ symmetric cells, ion conduction pathways provided by $3 \mathrm{D} \mathrm{COF}$ are beneficial for enhancing the ion transport and the $t^{+}$of 3D-Sp-COF approaches 0.7 . The value overwhelms most of the typical PEO-based polymer electrolytes and even comparable to the signal-ion electrolyte systems. Because of the large surface area and hollow spherical architecture, the 3D COF shows a high specific capacitance of $251 \mathrm{~F} \mathrm{~g}^{-1}$ at a current density of $0.5 \mathrm{~A} \mathrm{~g}^{-1}$, which is superior to the vast majority of $2 \mathrm{D}$ COFs and other porous electrode materials. Remarkably, the specific capacitance increases dramatically with the numbers of cycles, achieving a maximum of $364 \mathrm{~F} \mathrm{~g}^{-1}$ at the 6000th cycle due to the wetting activation of the hollow spheres. This study provides a feasible morphological modulation strategy to improve the electrochemical characteristics, which 
provides a deep insight into the design and synthesis of efficient and stable electrode materials for energy storage applications.

\section{Methods}

Materials. 2,2',7,7'-Tetrabromo-9,9'-spirobifluorene, 4-aminophenylboronic acid pinacol ester, terephthalaldehyde, and 2,5-dihydroxyterephthalaldehyde were purchased from Energy Chemical. All other reagents and solvents were commercially available and used as received.

Synthesis of 3D-Sp-COF. A Pyrex tube was charged with $4,4^{\prime}, 4^{\prime \prime}, 4^{\prime \prime \prime}-\left(9,9^{\prime}\right.$-spirobifluorene-2,2',7,7'-tetrayl)tetraaniline $(68.1 \mathrm{mg}, 0.1 \mathrm{mmol})$, terephthalaldehyde $(26.8 \mathrm{mg}, 0.2 \mathrm{mmol})$, and solvent mixture of 1,2-dichlorobenzene $/ n$-butanol $/ 6 \mathrm{M}$ aqueous acetic acid (7:3:1, by volume). This mixture was sonicated for $5 \mathrm{~min}$ in order to get a homogeneous dispersion. The tube was then flash frozen at $77 \mathrm{~K}$ (liquid $\mathrm{N}_{2}$ bath), degassed by freeze-pump-thaw technique for three times. The tube was sealed off and then placed in an oven at $120^{\circ} \mathrm{C}$ for a certain period of time $(6,12,24,48,72,96 \mathrm{~h})$. The resulting precipitate was filtered, washed with anhydrous tetrahydrofuran, and extracted by Soxhlet extractor for $24 \mathrm{~h}$. The solid was dried at $80^{\circ} \mathrm{C}$ under vacuum for $12 \mathrm{~h}$ to afford yellow powder $(63.0 \mathrm{mg}, 72 \%$ yield).

Synthesis of 3D-SpOH-COF. A Pyrex tube was charged with $4,4^{\prime}, 4^{\prime \prime}, 4^{\prime \prime \prime}-\left(9,9^{\prime}\right.$ spirobifluorene-2,2',7,7'-tetrayl)tetraaniline $(68.1 \mathrm{mg}, 0.1 \mathrm{mmol}), 2,5$-dihydroxyterephthalaldehyde $(33.2 \mathrm{mg}, 0.2 \mathrm{mmol})$, and solvent mixture of 1,2-dichlorobenzene $/ n$-butanol $/ 6 \mathrm{M}$ aqueous acetic acid (8:5:1, by volume). This mixture was sonicated for $5 \mathrm{~min}$ in order to get a homogeneous dispersion. The tube was then flash frozen at $77 \mathrm{~K}$ (liquid $\mathrm{N}_{2}$ bath), degassed by a freeze-pump-thaw technique for three times. The tube was sealed off and then placed in an oven at $120^{\circ} \mathrm{C}$ for a certain period of time $(12,24,48,72,96 \mathrm{~h})$. The resulting precipitate was filtered, washed with anhydrous tetrahydrofuran, and extracted by a Soxhlet extractor for $24 \mathrm{~h}$. The solid was dried at $80^{\circ} \mathrm{C}$ under vacuum for $12 \mathrm{~h}$ to afford orange powder (63.9 mg, 68\% yield).

\section{Data availability}

All data needed to evaluate the conclusions given in the paper are present in the Article and Supplementary Information. Any additional data related to this paper may be requested from the corresponding authors.

Received: 20 February 2020; Accepted: 19 August 2020;

Published online: 03 November 2020

\section{References}

1. Yuan, S. et al. Covalent organic frameworks for membrane separation. Chem. Soc. Rev. 48, 2665-2681 (2019).

2. Fan, H. et al. Covalent organic framework-covalent organic framework bilayer membranes for highly selective gas separation. J. Am. Chem. Soc. 140, 10094-10098 (2018)

3. Yan, S. et al. Three-dimensional salphen-based covalent-organic frameworks as catalytic antioxidants. J. Am. Chem. Soc. 141, 2920-2924 (2019).

4. Wang, X. et al. Sulfone-containing covalent organic frameworks for photocatalytic hydrogen evolution from water. Nat. Chem. 10, 1180-1189 (2018).

5. DeBlase, C. R. et al. $\beta$-Ketoenamine-linked covalent organic frameworks capable of pseudocapacitive energy storage. J. Am. Chem. Soc. 135 , 16821-16824 (2013).

6. Chen, X. et al. High-lithium-affinity chemically exfoliated $2 \mathrm{D}$ covalent organic frameworks. Adv. Mater. 31, 1901640 (2019).

7. Xiong, Y. et al. Ultrahigh responsivity photodetectors of 2D covalent organic frameworks integrated on graphene. Adv. Mater. 32, 1907242 (2020).

8. Keller, N. et al. Oligothiophene-bridged conjugated covalent organic frameworks. J. Am. Chem. Soc. 139, 8194-8199 (2017).

9. Kulkarni, R. et al. Real-time optical and electronic sensing with a $\beta$-amino enone linked, triazine-containing 2D covalent organic framework. Nat. Commun. 10, 3228 (2019).

10. Cui, W.-R. et al. Regenerable and stable sp2 carbon-conjugated covalent organic frameworks for selective detection and extraction of uranium. Nat. Commun. 11, 436 (2020).

11. Zwaneveld, N. A. et al. Organized formation of $2 \mathrm{D}$ extended covalent organic frameworks at surfaces. J. Am. Chem. Soc. 130, 6678-6679 (2008).

12. Gole, B. et al. Microtubular self-assembly of covalent organic frameworks. Angew. Chem. Int. Ed. Engl. 57, 846-850 (2018).

13. Kandambeth, S. et al. Self-templated chemically stable hollow spherical covalent organic framework. Nat. Commun. 6, 6786 (2015).
14. Long, J. et al. $\mathrm{Bi}_{2} \mathrm{MoO}_{6}$ nanobelts for crystal facet-enhanced photocatalysis. Small 10, 2791-2795 (2014).

15. Peng, Y. et al. Ultrathin two-dimensional covalent organic framework nanosheets: preparation and application in highly sensitive and selective DNA detection. J. Am. Chem. Soc. 139, 8698-8704 (2017).

16. Yang, Z. et al. Activatable semiconducting theranostics: simultaneous generation and ratiometric photoacoustic imaging of reactive oxygen species in vivo. Adv. Mater. 30, 1707509 (2018)

17. Mitra, S. et al. Targeted drug delivery in covalent organic nanosheets (CONs) via sequential postsynthetic modification. J. Am. Chem. Soc. 139, 4513-4520 (2017).

18. Strasser, P., Gliech, M., Kuehl, S. \& Moeller, T. Electrochemical processes on solid shaped nanoparticles with defined facets. Chem. Soc. Rev. 47, 715-735 (2018).

19. Wang, S. et al. Exfoliation of covalent organic frameworks into few-layer redox-active nanosheets as cathode materials for lithium-ion batteries. J. Am. Chem. Soc. 139, 4258-4261 (2017).

20. Haase, F. et al. Topochemical conversion of an imine-into a thiazole-linked covalent organic framework enabling real structure analysis. Nat. Commun. 9, 2600 (2018)

21. Nguyen, V. \& Grünwald, M. Microscopic origins of poor crystallinity in the synthesis of covalent organic framework COF-5. J. Am. Chem. Soc. 140, 3306-3311 (2018).

22. Halder, A. et al. Decoding the morphological diversity in two dimensional crystalline porous polymers by core planarity modulation. Angew. Chem. Int. Ed. 55, 7806-7810 (2016).

23. $\mathrm{Bi}$, S. et al. Two-dimensional semiconducting covalent organic frameworks via condensation at arylmethyl carbon atoms. Nat. Commun. 10, 2467 (2019).

24. $\mathrm{Ma}, \mathrm{T}$. et al. Single-crystal X-ray diffraction structures of covalent organic frameworks. Science 361, 48-52 (2018).

25. Ascherl, L. et al. Molecular docking sites designed for the generation of highly crystalline covalent organic frameworks. Nat. Chem. 8, 310-316 (2016).

26. Cote, A. P. et al. Porous, crystalline, covalent organic frameworks. Science 310, 1166-1170 (2005).

27. Banerjee, T. et al. Sub-stoichiometric $2 \mathrm{D}$ covalent organic frameworks from tri-and tetratopic linkers. Nat. Commun. 10, 2689 (2019).

28. Wei, S. et al. Semiconducting 2D triazine-cored covalent organic frameworks with unsubstituted olefin linkages. J. Am. Chem. Soc. 141, 14272-14279 (2019).

29. Tan, J. et al. Manipulation of amorphous-to-crystalline transformation: towards the construction of covalent organic framework hybrid microspheres with NIR photothermal conversion ability. Angew. Chem. Int. Ed. 55, 13979-13984 (2016).

30. Karak, S. et al. Constructing ultraporous covalent organic frameworks in seconds via an organic terracotta process. J. Am. Chem. Soc. 139, 1856-1862 (2017).

31. Beaudoin, D., Maris, T. \& Wuest, J. D. Constructing monocrystalline covalent organic networks by polymerization. Nat. Chem. 5, 830 (2013).

32. Evans, A. M. et al. Seeded growth of single-crystal two-dimensional covalent organic frameworks. Science 361, 52-57 (2018).

33. Matsumoto, M. et al. Lewis-acid-catalyzed interfacial polymerization of covalent organic framework films. Chem 4, 308-317 (2018).

34. Wang, H. et al. Recent progress in covalent organic framework thin films: fabrications, applications and perspectives. Chem. Soc. Rev. 48, 488-516 (2019).

35. Dey, K. et al. Selective molecular separation by interfacially crystallized covalent organic framework thin films. J. Am. Chem. Soc. 139, 13083-13091 (2017).

36. Yu, F. et al. Photostimulus-responsive large-area two-dimensional covalent organic framework films. Angew. Chem. Int. Ed. 58, 16101-16104 (2019).

37. Khayum, M. A. et al. Chemically delaminated free-standing ultrathin covalent organic nanosheets. Angew. Chem. Int. Ed. 55, 15604-15608 (2016).

38. Ying, Y. et al. Ultrathin two-dimensional membranes assembled by ionic covalent organic nanosheets with reduced apertures for gas separation. J. Am. Chem. Soc. 142, 4472-4480 (2020).

39. Zhu, H.-J. et al. Efficient electron transmission in covalent organic framework nanosheets for highly active electrocatalytic carbon dioxide reduction. Nat. Commun. 11, 497 (2020).

40. El-Mahdy, A. F. et al. A hollow microtubular triazine-and benzobisoxazolebased covalent organic framework presenting sponge-like shells that functions as a high-performance supercapacitor. Chem. Asian J. 14, 1429-1435 (2019).

41. Lu, Z. et al. A hollow microshuttle-shaped capsule covalent organic framework for protein adsorption. J. Mater. Chem. B 7, 1469-1474 (2019).

42. Wan, S. et al. A belt-shaped, blue luminescent, and semiconducting covalent organic framework. Angew. Chem. Int. Ed. 47, 8826-8830 (2008).

43. Huang, W. et al. Solvothermal synthesis of microporous, crystalline covalent organic framework nanofibers and their colorimetric nanohybrid structures. ACS Appl. Mater. Interfaces 5, 8845-8849 (2013). 
44. Wang, S. et al. Reversible polycondensation-termination growth of covalentorganic-framework spheres, fibers, and films. Matter 1, 1592-1605 (2019).

45. Ma, W. et al. Size-controllable synthesis of uniform spherical covalent organic frameworks at room temperature for highly efficient and selective enrichment of hydrophobic peptides. J. Am. Chem. Soc. 141, 18271-18277 (2019).

46. Sasmal, H. S. et al. Covalent self-assembly in two dimensions: connecting covalent organic framework nanospheres into crystalline and porous thin films. J. Am. Chem. Soc. 141, 20371-20379 (2019).

47. Baldwin, L. A., Crowe, J. W., Pyles, D. A. \& McGrier, P. L. Metalation of a mesoporous three-dimensional covalent organic framework. J. Am. Chem. Soc. 138, 15134-15137 (2016).

48. Zhang, Y. et al. Three-dimensional anionic cyclodextrin-based covalent organic frameworks. Angew. Chem. Int. Ed. 56, 16313-16317 (2017).

49. Liu, Y. et al. 3D covalent organic frameworks of interlocking $1 \mathrm{D}$ square ribbons. J. Am. Chem. Soc. 141, 677-683 (2018).

50. Lin, G. et al. 3D porphyrin-based covalent organic frameworks. J. Am. Chem. Soc. 139, 8705-8709 (2017).

51. Ma, Y.-X. et al. A dynamic three-dimensional covalent organic framework. J. Am. Chem. Soc. 139, 4995-4998 (2017).

52. Ding, H. et al. An AIEgen-based 3D covalent organic framework for white light-emitting diodes. Nat. Commun. 9, 5234 (2018).

53. Wu, C. et al. Highly conjugated three-dimensional covalent organic frameworks based on spirobifluorene for perovskite solar cell enhancement. $J$. Am. Chem. Soc. 140, 10016-10024 (2018).

54. Ghosh, A., Wang, C. \& Kofinas, P. Block copolymer solid battery electrolyte with high Li-ion transference number. J. Electrochem. Soc. 157, A846-A849 (2010).

55. Zhou, W. et al. Plating a dendrite-free lithium anode with a polymer/ceramic/ polymer sandwich electrolyte. J. Am. Chem. Soc. 138, 9385-9388 (2016).

56. Zhou, D. et al. Stable conversion chemistry-based lithium metal batteries enabled by hierarchical multifunctional polymer electrolytes with near-single ion conduction. Angew. Chem. Int. Ed. 58, 6001-6006 (2019).

57. Zhang, H. et al. Single lithium-ion conducting solid polymer electrolytes: advances and perspectives. Chem. Soc. Rev. 46, 797-815 (2017).

58. Watanabe, M., Tokuda, H. \& Muto, S. Anionic effect on ion transport properties in network polyether electrolytes. Electrochim. Acta 46, 1487-1491 (2001).

59. Mao, B. et al. Solubility-parameter-guided solvent selection to initiate Ostwald ripening for interior space-tunable structures with architecturedependent electrochemical performance. Angew. Chem. Int. Ed. 57, 446-450 (2018).

60. Xie, J. et al. Intralayered Ostwald ripening to ultrathin nanomesh catalyst with robust oxygen-evolving performance. Adv. Mater. 29, 1604765 (2017).

61. Shen, L. et al. Self-templated formation of uniform $\mathrm{NiCo}_{2} \mathrm{O}_{4}$ hollow spheres with complex interior structures for lithium-ion batteries and supercapacitors. Angew. Chem. Int. Ed. 54, 1868-1872 (2015).

62. Tian, X. L. et al. Nearly monodisperse ferroelectric $\mathrm{BaTiO}_{3}$ hollow nanoparticles: size-related solid evacuation in ostwald-ripening-lnduced hollowing process. Cryst. Growth Des. 10, 3990-3995 (2010).

63. Liu, B. \& Zeng, H. C. Symmetric and asymmetric Ostwald ripening in the fabrication of homogeneous core-shell semiconductors. Small 1, 566-571 (2005).

64. Li, X.-C. et al. Redox-active triazatruxene-based conjugated microporous polymers for high-performance supercapacitors. Chem. Sci. 8, 2959-2965 (2017).

65. Zhang, Y. Z. et al. A simple approach to boost capacitance: flexible supercapacitors based on manganese oxides@ MOFs via chemically induced in situ self-transformation. Adv. Mater. 28, 5242-5248 (2016).

66. Zhang, Y.-Z. et al. Printed supercapacitors: materials, printing and applications. Chem. Soc. Rev. 48, 3229-3264 (2019).

67. Sun, J. et al. A molecular pillar approach to grow vertical covalent organic framework nanosheets on graphene: hybrid materials for energy storage. Angew. Chem. Int. Ed. 57, 1034-1038 (2018).

68. Sheberla, D. et al. Conductive MOF electrodes for stable supercapacitors with high areal capacitance. Nat. Mater. 16, 220 (2017).
69. Halder, A. et al. Interlayer hydrogen-bonded covalent organic frameworks as high-performance supercapacitors. J. Am. Chem. Soc. 140, 10941-10945 (2018).

\section{Acknowledgements}

We acknowledge financial support from the National Natural Science Foundation of China (21835003, 91833304, 21674050, 21422402, 61704077, 62005126), the National Key Basic Research Program of China (973 Program, 2017YFB0404501, 2014CB648300), the Natural Science Foundation of Jiangsu Province (BE2019120), the China Postdoctoral Science Foundation (2019M650121, 2016M601784, 2017M620519, 2017T100358), the Postdoctoral Science Foundation of Jiangsu Province (1701135B), Program for Jiangsu Specially-Appointed Professor (RK030STP15001), the Six Talent Peaks Project of Jiangsu Province (TD-XCL-009), the 333 Project of Jiangsu Province (BRA2017402), the NUPT "1311 Project" and the NUPT Scientific Foundation (NY219021, NY218164, NY217169), the Leading Talent of Technological Innovation of National Ten-Thousands Talents Program of China, the Excellent Scientific and Technological Innovative Teams of Jiangsu Higher Education Institutions (TJ217038), the Synergetic Innovation Center for Organic Electronics and Information Displays, and the Priority Academic Program Development of Jiangsu Higher Education Institutions (PAPD).

\section{Author contributions}

Y.-Y.L. and X.-C.L. contributed to the material synthesis, theoretical calculation, and the mechanism analysis. S.W. performed X-ray diffraction and chronoamperometry measurements. T.C. and C.L. performed CV and GCD measurements. H.Y. performed TEM measurements. Y.G. performed TGA and FT-IR measurements. W.-Y.L. and W.H. contributed to overall experimental design and supervised the project. Y.-Y.L., X.-C.L., and W.-Y.L. wrote the manuscript. All authors discussed the results and commented on the manuscript.

\section{Competing interests}

The authors declare no competing interests.

\section{Additional information}

Supplementary information is available for this paper at https://doi.org/10.1038/s41467 020-18844-4.

Correspondence and requests for materials should be addressed to W.-Y.L. or W.H.

Peer review information Nature Communications thanks the anonymous reviewer(s) for their contribution to the peer review of this work.

Reprints and permission information is available at http://www.nature.com/reprints

Publisher's note Springer Nature remains neutral with regard to jurisdictional claims in published maps and institutional affiliations.

Open Access This article is licensed under a Creative Commons Attribution 4.0 International License, which permits use, sharing, adaptation, distribution and reproduction in any medium or format, as long as you give appropriate credit to the original author(s) and the source, provide a link to the Creative Commons license, and indicate if changes were made. The images or other third party material in this article are included in the article's Creative Commons license, unless indicated otherwise in a credit line to the material. If material is not included in the article's Creative Commons license and your intended use is not permitted by statutory regulation or exceeds the permitted use, you will need to obtain permission directly from the copyright holder. To view a copy of this license, visit http://creativecommons.org/ licenses/by/4.0/

(C) The Author(s) 2020 